VOL. 52 (1995) [253-261]

\title{
SOLUBLE AND LINEAR REPETITIVE GROUPS
}

\section{A.V. Kelarev and P.V. Shumyatsky}

Our main theorem describes FC-soluble and linear repetitive groups. As a corollary, we characterise algebraic linear repetitive semigroups.

\section{INTRODUCTION}

Let $S$ be a semigroup, and let $s=s_{1}, \ldots, s_{m}$ be a sequence of elements of $S$. A $k$-factorisation of $s$ is a sequence $t=t_{1}, \ldots, t_{m}$, where the $t_{j}$ 's are the values of $k$ consecutive segments of $s$, that is, $t_{j}=s_{i_{j}} s_{i_{j}+1} \cdots s_{i_{j+1}-1}$ for $j=1, \ldots, k$ and some $1 \leqslant i_{1}<i_{2}<\ldots<i_{k+1} \leqslant m+1$. We say that $t$ is a power $k$-factorisation if $t_{1}=\ldots=t_{k}$. A semigroup $S$ is said to be repetitive if and only if, for each finite subset $X$ of $S$ and every integer $k>0$, there exists a positive integer $L=L(S, X, k)$ such that every sequence $s_{1}, \ldots, s_{L}$ of elements of $X$ has a power $k$-factorisation.

Repetitive semigroups were introduced by Justin $[5,6]$. The fact that the infinite cyclic group is repetitive is a generalisation of the van der Waerden theorem on arithmetic progressions (see [6]). A well-known corollary to Ramsey's theorem implies that every finite semigroup is repetitive (see $[15$, Section 4.1$]$ ). Repetitivity is related to a few other combinatorial conditions (see $[9,12,13,15$, Section $4.2,16]$ ).

Complete descriptions of repetitive commutative semigroups and repetitive Rees quotients of free monoids were obtained in [7] and [10], respectively. The preservation of repetitivity by various constructions was investigated in [5], [6] and [17]. It is known that a nilpotent group is repetitive if and only if it is of linear growth $($ see $[4,6,8]$ and the survey [11]). In view of Gromov's theorem, this gives a description of all repetitive groups of polynomial growth. However, the problem of characterising repetitive groups remains open.

In this paper we solve this problem for FC-soluble and linear groups, obtaining a description in the language of forbidden sections. This result is analogous to Justin's characterisation of repetitive commutative semigroups (see Proposition 3.1 below). Note that FC-groups play key roles in considerations of several other combinatorial properties of groups (see, for example, [2]). The exact analog of our theorem is not true for arbitrary groups (Example 2.3).

Received 23rd November, 1994

Copyright Clearance Centre, Inc. Serial-fee code: 0004-9729/95 SA2.00+0.00. 
It was conjectured in [11] that every repetitive group has a linear growth. Our main theorem shows that this conjecture is true for FC-soluble and linear groups. On the other hand, there exist repetitive semigroups with non-linear growth ([10, Theorems 1 , $3]$, or [5]).

As an application of our main theorem we shall easily describe all algebraic linear repetitive semigroups (Corollary 2.2).

\section{Main Results}

A group $H$ is called a section of $G$ if it is a homomorphic image of a subgroup of $G$. We shall call a semigroup $S$ a section of $G$ if it is a homomorphic image of a subsemigroup of $G$. We shall use $\mathbb{Z}$ and $\mathbb{N}$ to denote the additive group of all integers and the additive semigroup of all positive integers respectively. Recall that the free commutative group (semigroup) of rank two is isomorphic to $\mathbb{Z} \times \mathbb{Z}$ (respectively, $\mathbb{N} \times \mathbb{N}$ ). For a subset $X$ of a semigroup $S$, put $X^{[n]}=\bigcup_{i=1}^{n} X^{i}$. As usual the wreath product of groups $G$ and $H$ is denoted by $G \backslash H$. The subgroup generated in $G$ by a set $X$ is denoted by $\langle X\rangle$.

Theorem 2.1. Let $G$ be an $F C$-soluble or linear group. Then the following are equivalent:

(i) $G$ is repetitive;

(ii) $G$ has linear growth;

(iii) every finitely generated subgroup of $G$ is cyclic-by-finite;

(iv) G has no section isomorphic to the free commutative semigroup of rank two.

COROLlary 2.2. An algebraic linear semigroup is repetitive if and only if it does not contain a subsemigroup that can be homomorphically mapped onto the free commutative semigroup of rank two.

EXAMPLE 2.3. Let $G$ be the infinite finitely generated Burnside group of Novikov and Adjan (see [1]) satisfying the identity $x^{n}=1$ for some $n$. Obviously, it has no section isomorphic to $\mathbb{N} \times \mathbb{N}$. However, it is not repetitive. Indeed, repetitivity and the identity $x^{n}=1$ easily imply that $G$ is locally finite, giving a contradiction. Thus, the analog of our main theorem for arbitrary groups is not true.

EXAMPLE 2.4. Let $C=\langle c\rangle$ be the infinite cyclic group with a generator $c$, and let $C_{i}=\left\langle c_{i}\right\rangle$ be the cyclic group of order $i$ with a generator $c_{i} ; i=2,3, \ldots$ Denote by $D$ the direct product of all these cyclic groups. We shall use additive notation for the group operation in $D$. Let $b_{i}$ denote the automorphism of $D$ which maps $c$ to $c+c_{i}$ and leaves all $c_{i}$ 's fixed. Put $B=\left\langle b_{i} ; i=2,3, \ldots\right\rangle$ and consider the semidirect 
product $G=D B$. Every finitely generated subgroup of $G$ is cyclic-by-finite, and so repetitive. However, $G$ has no normal infinite cyclic subgroup. Thus, $G$ is a repetitive group which is not an extension of a cyclic group by a locally finite group.

EXAMPLE 2.5. For a prime $p$, consider the wreath product $W=\mathbb{Z}_{p} \mid \mathbb{Z}$. It has no section isomorphic to the free Abelian group of rank two. By Lemma 3.8 below $W$ is not repetitive. Therefore in condition (iv) of the main theorem we cannot replace sections isomorphic to the free commutative semigroup of rank two by sections isomorphic to the free Abelian group of rank two.

\section{Proofs}

Our proofs rely on the technique develcped by Justin in other results concerning repetitivity. In particular, we shall use the following

Proposition 3.1. (Justin [7]). A commutative semigroup is repetitive if and only if it does not contain the free commutative semigroup of rank two.

For preliminaries on semigroups, algebraic linear semigroups, groups, FC-groups and linear groups we refer to $[3,17,18,20]$ and $[21]$, respectively. Let us first record two easy lemmas.

Lemma 3.2. A semigroup is repetitive if and only if all its finitely generated subsemigroups are repetitive.

LEMмA 3.3. The class of repetitive semigroups is closed under subsemigroups and homomorphic images.

The class of repetitive groups is not closed under group extensions, as is clear from Example 2.5. In contrast, the next lemma shows that the class of repetitive semigroups is closed under ideal extensions.

Lemma 3.4. Let $T$ be an ideal of a semigroup $S$. If $T$ and $S / T$ are repetitive, then $S$ is repetitive, too.

Proof: Let $S$ be a semigroup, $T$ an ideal of $S$, and let $T$ and $S / T$ be repetitive. Take any finite subset $X$ of $S$. We shall denote the image of $X$ in $S / T$ by the same letter $X$. Take a positive integer $k$.

Put $m=L(S / T, X, k), Y=X^{m} \cap T, n=L(T, Y, k)$, and $L=m n$. We claim that $L$ satisfies the definition of $L(S, X, k)$.

Consider a sequence $s=s_{1}, \ldots, s_{L}$ of elements of $X$ and its factorisation $u=$ $u_{1}, \ldots, u_{n}$, where $u_{i}=s_{(i-1) m+1} \cdots s_{i m}$ for $i=1, \ldots, n$. If $u_{1}, \ldots, u_{n} \in T$, then $u$ has a power $k$-factorisation by the choice of $n$. If, however, $u_{i} \notin T$ for some $i$, then $u_{i}$ has a power $k$-factorisation by the choice of $m$. The latter is a power $k$-factorisation of $s$, too. Thus $S$ is repetitive. 
LEMma 3.5. If a semigroup $S$ is the union of its repetitive right (or left) ideals $R_{i}$, where $i \in I$, then $S$ is repetitive.

Proof: We shall consider only the case of right ideals, since for left ideals the proof is similar. Every finitely generated subsemigroup of $S$ is the union of a finite number of subsemigroups of the $R_{i}$ 's. All these subsemigroups are repetitive. In view of Lemma 3.2 without loss of generality we may assume that the indexing set $I$ is finite. By induction it suffices to consider the case where $|I|=2$.

Take an arbitrary finite subset $X$ of $S$. Let $X_{i}=X \cap R_{i}$, for $i=1,2$. Put $m=L\left(R_{2}, X_{2}, k\right)$ and $Y_{1}=X_{1} \cup X_{1} X^{[m]}$. Since $Y_{1}$ is contained in the repetitive right ideal $R_{1}$, the number $L\left(R_{1}, Y_{1}, k\right)$ can be defined, and we put $n=m\left(L\left(R_{1}, Y_{1}, k\right)+1\right)$. We claim that $n$ can serve as $L(S, X, k)$.

Indeed, look at an arbitrary sequence $s=s_{1}, \ldots, s_{n}$ of elements of $X$. Let $s_{i_{1}}, s_{i_{2}}, \ldots, s_{i_{\ell}}$ be all elements of $s$ belonging to $X_{1}$, where $i_{1}<i_{2}<\ldots<i_{\ell}$.

If $s$ has $m$ consecutive elements which all belong to the set $X_{2}$, then the segment formed by these elements has a power $k$-factorisation by the choice of $m$.

It remains to consider the case where every segment of $m$ consecutive elements of $s$ contains an element of $X_{1}$. Then $i_{1} \leqslant m$, and $i_{j+1} \leqslant i_{j}+m$, for all $j=1, \ldots, \ell-1$. Hence by the choice of $n$ we get $\ell>L\left(R_{1}, Y_{1}, k\right)$. Since $R_{1}$ is a right ideal, all elements $u_{j}=s_{i_{j}} \cdots s_{i_{j+1}-1}$ of the factorisation $u=u_{1}, \ldots, u_{\ell-1}$ belong to $Y_{1}$. Therefore $s$ has a power $k$-factorisation again, because $\ell-1 \geqslant L\left(R_{1}, Y_{1}, k\right)$. This completes the proof.

REMARK 3.6. It follows from Lemmas $3.2,3.3$ and 3.4, that every semigroup $S$ with zero has a largest repetitive ideal $\mathcal{R}(S)$ such that $S / \mathcal{R}(S)$ has no nonzero repetitive ideals.

LEMMA 3.7. If $G$ is a repetitive group, and $S=M^{0}(G ; I, \Lambda ; P)$ is a Rees matrix semigroup, then $S$ is repetitive.

Proof: It is well-known that every Rees matrix semigroup $S$ is the union of its right ideals $R_{i}, i \in I$, where every $R_{i}$ is the union of its left ideals $H_{i \lambda}, \lambda \in \Lambda$, such that each $H_{i \lambda}$ is either a group isomorphic to $G$ with zero adjoined, or a semigroup with zero multiplication [3, Section 3.2]. Applying Lemma 3.5 twice we see that $S$ is repetitive.

LEMMA 3.8. If $G$ is a nontrivial group and $H$ is a nonperiodic group, then the wreath product $W=G$ । $H$ contains free semigroups of all finite ranks.

Proof: The base group $B$ of $W$ is the direct product $B=\prod_{h \in H} B_{h}$, where each $B_{h}$ is a copy of $G$. Denote the image of an element $g \in G$ in $B_{h}$ by $g_{h}$. Fix any $g \in G \backslash\{e\}$ and any element of infinite order $h \in H \backslash\{e\}$. Consider elements $x_{1}=\left(h^{2}, g_{h}\right)$ and $x_{2}=\left(h^{2}, g_{h^{2}}\right)$. 
The product of elements $\left(a, b_{q}\right)\left(c, d_{h}\right)$, where $b, d \in G, a, c, q, h \in H$, is equal to $\left(a c, b_{q c} d_{h}\right)$. Therefore, for any $i_{1}, i_{2}, \ldots, i_{n} \in\{1,2\}$, the product $y=x_{i_{1}} x_{i_{2}} \cdots x_{i_{n}}$ is equal to $\left(h^{2 n}, g_{k_{1}} g_{k_{2}} \cdots g_{k_{n}}\right)$, where $k_{j}=h^{t_{j}}$ and $t_{j}=i_{j}+2(n-j)$ for $j=1,2, \ldots, n$. Since the numbers $t_{1}, \ldots, t_{n}$ are pairwise distinct, all elements $g_{k_{1}}, \ldots, g_{k_{n}}$ belong to different components of the direct product $B$. Therefore, the sequence $i_{1}, \ldots, i_{n}$ is uniquely determined by the resulting product $y$. Thus $x_{1}$ and $x_{2}$ generate a free semigroup of rank two. The latter contains free subsemigroups of all finite ranks.

Lемма 3.9. If a group $G$ contains a cyclic subgroup of finite index, then it is cyclic-by-finite.

Proof: Let $H$ be the cyclic subgroup of $G$. Given that $H$ is of finite index in $G$, the core $H_{G}$ also has a finite index in $G[18,1.6 .9]$. Obviously, $H_{G}$ is a cyclic normal subgroup of $G$.

Lemma 3.10. Let $G$ possess an infinite cyclic subgroup $H$ such that $G / H$ is infinite cyclic. Then $G$ has a subgroup isomorphic to the free Abelian group of rank two.

Proof: Let $g H$ generate the cyclic group $G / H$. Since the automorphism group of $H$ is of order two, $g^{2} \in C_{G}(H)$. It follows that $\left\langle g^{2}, H\right\rangle$ is isomorphic to the free Abelian group of rank two.

Lemma 3.11. A finite-by-cyclic group is cyclic-by-finite.

Proof: Let $N$ be finite and $G / N$ cyclic, generated by $h N$. Then $\langle h\rangle=H$ is an infinite cyclic subgroup of $G$. Since $G=\bigcup_{m=1}^{\infty} h^{m} N$, we get $G=\bigcup_{x \in N} H x$. Thus $|G: H|$ is finite. The result follows by Lemma 3.9 .

LEмMA 3.12. A finitely generated FC-group has no section isomorphic to the free group of rank two if and only if it is cyclic-by-finite.

Proof: By [20], Corollary 1.5, $G$ is finite over its centre (see also $[18,14.5 .10]$ ). Denote by $C$ the centre of $G$. Since $C$ has finite index in $G$, it follows that $C$ is finitely generated $[18,4.2 .10]$. Therefore it is the direct product of finitely many cyclic groups $[18,4.2 .10]$. Given that $G$ has no section isomorphic to the free Abelian group of rank two, we see that $C$ is cyclic-by-finite. Therefore $G$ contains a cyclic subgroup of finite index. Lemma 3.9 completes the proof.

LEMMA 3.13. If an FC-group $G$ has no section isomorphic to $\mathbb{Z} \times \mathbb{Z}$, then it is an extension of a locally finite group by a cyclic group.

Proof: Consider the set $T$ of all periodic elements of $G$. By $[18,14.5 .9] T$ is a normal locally finite subgroup of $G$, and the quotient group $G / T$ is an Abelian torsionfree group. Given that $G$ has no sections isomorphic to $\mathbb{Z} \times \mathbb{Z}$, the same can be said 
of $G / T$. Therefore $G / T$ is cyclic.

LemmA 3.14. Let $G=\langle a, b\rangle$ be a group without sections isomorphic to $\mathbb{N} \times \mathbb{N}$. Suppose that the normal closure $T$ of $a$ in $G$ is an FC-group. Then $G$ is cyclic-byfinite.

Proof: Let $F$ be the set of all elements of finite order in $T$. By Lemma $3.13 F$ is locally finite and $T / F$ is cyclic.

First, assume that $a$ is of infinite order. Then $T / F$ is infinite cyclic. Suppose that $G / T$ is infinite. Since $F$ is normal in $G$, we can factor it out and get a torsion free cyclic-by-cyclic group. By Lemma 3.10 it has a section isomorphic to $\mathbb{N} \times \mathbb{N}$, which is also a section of $G$. This contradiction shows that $G / T$ is finite. Then $T$ is finitely generated by $[18,1.6 .11]$. Lemma 3.12 tells us that $T$ is cyclic-by-finite. By Lemma 3.9 , the same can be said of $G$. Thus, if $a$ is of infinite order, then $G$ is cyclic-by-finite.

Now, we assume that $a$ is of finite order. Then $a \in F$, and so $T=F$ is locally finite. Let $A$ be the normal closure of $a$ in $T$. It is finite, because $T$ is a locally finite and normal group by $[18,14.5 .8]$. We proceed by induction on $|A|$.

The case where $|A|=1$ is trivial, since then $a$ is equal to the identity of $G$, and $G$ is cyclic. Assume that $|A|>1$.

First, consider the case where an element $x$ of $A$ belongs to the FC-centre of $G$. Then the the normal closure $N$ of $x$ in $G$ is finite. By the induction assumption $G / N$ is cyclic-by-finite. Lemma 3.9 implies that $G$ is cyclic-by-finite.

Second, consider the case where no element of $A$ is contained in the FC-centre of $G$. Let $B=\langle b\rangle$. If $C_{B}(y) \neq 1$ for some $y \in A$, then $b^{k}$ commutes with $y$ for some $k$, and then $y^{G}=y^{T} \cup y^{T b} \cup \ldots \cup y^{T b^{k-1}}$ is finite, that is, $y$ is in the FC-centre of $G$. Therefore, in this case $C_{B}(y)=1$ for all $y \in A$, and $B$ is infinite.

Let now $D$ be some subgroup of $A$ that is minimal normal in $T$. Take any positive integer $k$. Since the intersection of $D$ and any of its conjugates is again normal, by the minimality of $D$ it follows that $D \cap b^{-k} D b^{k}$ equals $D$ or 1 . In the former case, however, we get a contradiction. Indeed, some element of $B$ normalises $D$, and so the normaliser of $D$ in $G$ has finite index. Since $D$ is finite, the centraliser of $D$ has a finite index in $G$. Therefore $B$ is contained in the FC-centre of $G$, a contradiction. Thus $D \cap b^{-k} D b^{k}=1$ for all $k$. It is well-known and easily verifed that if two normal subgroups have trivial intersection then they commute. Therefore $\left[D, b^{-k} D b^{k}\right]=1$.

Choose an element $d$ of prime order $p$ in $D$, and put $d_{i}=b^{-i} d b^{i} ; i \in \mathbb{Z}$. Then the group $M=\left\langle d_{i} ; i \in \mathbb{Z}\right\rangle$ is Abelian of prime exponent $p$. We view $M$ as a $B$-module over the field of order $p$. If the system $\left\{d_{i} ; i \in \mathbb{Z}\right\}$ is not linearly independent then there exists a positive integer $j$ such that

$$
d_{j} \in\left\langle d_{i} ; 0 \leqslant i \leqslant j-1\right\rangle=V
$$


Then $V$ is a finite $B$-submodule. This gives a contradiction because $C_{B}(d)=1$. Therefore the system $\left\{d_{i} ; i \in \mathbb{Z}\right\}$ is linearly independent. Hence the group $M$ is the direct product of $\left\langle d_{i}\right\rangle$ 's. These cyclic groups are naturally indexed by the elements from $B$, and $B$ acts on $M$ as in the wreath product $\langle d\rangle\langle B$. Thus the group $M B$ is isomorphic to the wreath product $\langle d\rangle \mid B$. This contradicts Lemma 3.8 and completes the proof.

LemMa 3.15. Let $G$ be a finitely generated $F C$-soluble group without sections isomorphic to $\mathbb{N} \times \mathbb{N}$. Then $G$ is cyclic-by-finite.

Proof: Let $1=T_{0} \leqslant T=T_{1} \leqslant \ldots \leqslant T_{k}=G$ be the normal series of smallest length in $G$ such that $T_{i} / T_{i-1}$ is an $F C$-group for $i=1,2, \ldots$ We use induction on $k$. If $k=1$ then $G$ is a finitely generated $F C$-group and so Lemma 3.12 tells us that $G$ is cyclic-by-finite.

Let now $k \geqslant 2$. By the inductive assumption $G / T$ is cyclic-by-finite. Let $C$ be a cyclic subgroup in $\mathrm{G}$ such that $T C / T$ is of finite index in $G / T$. Then $T C$ is finitely generated as it has finite index in a finitely generated group. If $T C / T$ is finite then $T$ is of finite index in $G$ and therefore is finitely generated. By Lemma 3.12 and Lemma $3.9 G$ is cyclic-by-finite.

Thus assume that $T C / T$ is infinite. Since $T C$ is finitely generated, there exist finitely many elements $t_{1}, t_{2}, \ldots, t_{r} \in T$ such that $G=\left\langle t_{1}, t_{2}, \ldots, t_{r}, C\right\rangle$. By Lemma 3.14 each of subgroups $\left\langle t_{i}, C\right\rangle$ is cyclic-by-finite. It follows that each $t_{i}$ centralises a subgroup of finite index in $C$. Let $D$ be the intersection of the centralisers of all $t_{i}$ 's in $C$. Then $D$ is of finite index in $C$ and $D \leqslant Z(T C)$. If $T$ is not periodic then the subgroup $Z(T) D$ possesses a subgroup isomorphic to $\mathbb{Z} \times \mathbb{Z}$ [20, Theorem 1.7]. Assume that $T$ is periodic. Then so is $T C / D$. Evidently $T C / D$ is locally finite and therefore it is finite. It follows that $D$ has finite index in $G$, and by Lemma 3.9 the proof is complete.

Proof of Theorem 2.1: Clearly, all the conditions (i) to (iv) hold in $G$ if and only if they are valid in all finitely generated subgroups of $G$. Therefore it suffices to prove the theorem for a finitely generated group $G$.

Proposition 3.1 and Lemma 3.3 show that (i) implies (iv). The implication (iii) $\Rightarrow$ (ii) is obvious. The equivalence of (i) and (ii) for linear groups was proved in [8].

It is known that if a group $S$ of linear growth is generated by a finite set $X$, then there exists a finite subset $F$ of $S$ such that every element of $S$ can be written in the form $u v^{n} w$ with $u, v, w \in F$ and $n \in \mathbb{N}$ (see [4] or [10], the proof of Theorem 3, or [14], the proof of Lemma 2.4). It easily follows that every group of linear growth is repetitive. Thus (ii) implies (i).

It remains to prove the implication (iv) $\Rightarrow$ (iii). If $G$ is FC-soluble, then Lemma 
3.15 proves the claim. If $G$ is linear, then [19, Corollary 1] tells us that either $G$ is soluble-by-finite or it contains a free subgroup. It follows that $G$ has a soluble normal subgroup $N$ of finite index. Applying Lemma 3.15 to $N$, we see that $G$ is cycic-by-finite again. This proves the result.

Proof of Corollary 2.2: If a semigroup is repetitive, then, as above, it has no sections isomorphic to the free commutative semigroup.

Conversly, assume that $S$ has no such sections. For proving that $S$ is repetitive, it suffices to consider the case where $S$ has zero, since we can adjoin a zero to $S$. Every algebraic linear semigroup with zero has a finite ideal chain with nilpotent of completely 0 -simple factors [17, Remark 3.29]. These factors are isomorphic to Rees matrix semigroups [3, Section 3.2]. Obviously, every nilpotent semigroup is repetitive. Therefore, Lemmas 3.4, 3.7 and Theorem 2.1 complete the proof.

\section{REFERENCES}

[1] S.I. Adjan, The Burnside problem and identities in groups (Springer-Verlag, Berlin, Heidelberg, New York, 1978).

[2] R.D. Blyth and A.H. Rhemtulla, 'Rewritable products in $\mathrm{FC}$-by-finite groups', Canad. J. Math. 41 (1989), 369-384.

[3] J.M. Howie, An introduction to semigroup theory (Academic Press, New York, 1976).

[4] J. Justin, 'Groupes et semi-groupes à croissance linéaire", C. R. Acad. Sci. Paris 273 (1971), 212-214.

[5] J. Justin, 'Sur une construction de Bruck et Reilly', Semigroup Forum 3 (1971), 148-155.

[6] J. Justin, 'Généralisation du théorème de van der Waerden sur les semi-groupes répétitifs', J. Combin. Theory Ser. A. 12 (1972), 357-367.

[7] J. Justin, 'Characterization of the repetitive commutative semigroups', J. Algebra 21 (1972), 87-90.

[8] J. Justin, 'Groupes linéaires répétitifs', C.R. Acad. Sci. Paris 292 (1981), 349-350.

[9] J. Justin and G. Pirillo, 'Une condition de finitude pour les semi-groupes finiment engendrés', C.R. Acad. Sci. Paris 306 (1988), 63-65.

[10] J. Justin and G. Pirillo, 'Factorial languages and some combinatorial properties of semigroups', Internat. J. Algebra Comput. 3 (1993), 295-316.

[11] J. Justin and G. Pirillo, 'On some questions and conjectures in combinatorial semigroup theory Southeast Asian Bull. Math.' 18, pp. 91-104.

[12] J. Justin, G. Pirillo and S. Varricchio, 'Unavoidable regularities and finiteness conditions for semigroups', in Theoretical Computer Science, (Bertoni, Böhm and Migliolo, Editors), Proceedings of the Third Italian Conference Mantova, 2-4 November 1989 (World Scientific, 1989).

[13] A.V. Kelarev, 'Combinatorial properties and homomorphisms of semigroups', Internat. J. Algebra Comput. (to appear). 
[14] G.R. Krause and T.H. Lenagan, Growth of algebras and Gelfand-Kirillov dimension, Research Notes 116 (Pitman, Boston, 1985).

[15] M. Lothaire, Combinatorics on words (Addison-Wesley, London, 1983).

[16] G. Pirillo, 'On a finiteness condition for a finitely generated semigroup', Rend. Circ. Math. Palermo 238 (1989), 212-216.

[17] M.S. Putcha, Linear algebraic monoids, London Math. Soc. Lect. Notes Ser. 133 (Cambridge University Press, Cambridge, 1988).

[18] D.J.S. Robinson, $A$ course in the theory of groups (Springer-Verlag, Berlin, Heidelberg, New York, 1982)).

[19] J. Tits, 'Free subgroups in linear groups', J. Algebra 20 (1972), 250-270.

[20] M.J. Tomkinson, FC-groups (Pitman, London, 1984).

[21] B.A.F. Wehrfritz, Infinite linear groups (Springer-Verlag, Berlin, Heidelberg, New York, 1973).

Department of Mathematics

The University of Tasmania

Hobart, Tas 7001

Australia
Department of Mathematics

Technion - Israel Institute of Technology

32000 Haifa

Israel 